\title{
Performans ng mga Gurong Lesbian, Gay, Bisexual, Transgender, Queer (LGBTQ+) sa pagtuturo ng akademikong Filipino
}

\author{
Gelilio, Eric $\square$ \\ Gallanosa National High School, Philippines (gelilioeric@gmail.com)
}

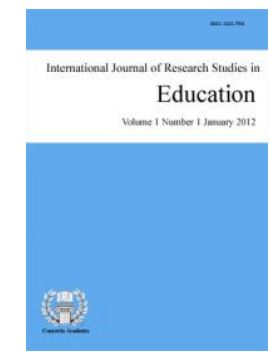

ISSN: 2243-7703 Online ISSN: 2243-7711

Received: 14 October 2021

Revised: 1 October 2021 DOI: $10.5861 /$ ijrse.2021.a120

Accepted: 20 October 2021

OPEN ACCESS

\section{Abstract}

The study was conceptualized to assess the performance of LGBTQ+ teachers in teaching Filipino discipline in the Division of Sorsogon Province particular in Secondary level of Education, School year 2019-2020. Research utilized descriptive analysis design. Purposive sampling was used to gather the data from 1,252 respondents in 22 Secondary schools in Sorsogon Province division. Frequency count, percentage and ranking was employed to analyze the impacts, factors, and performance of LGBTQ+ teachers teaching Filipino subject. Based on analysis of data, LGBTQ+ teachers converged on the higher order of comprehension through their innovations and strategies in teaching language and literature. According to Sandoval (2019), new developed teaching strategy is imperative to ensure the learning progress. Hence, it is important that teachers recreate and enhanced their teaching strategies for the lessons to be enticing for their students. Based on the study results, lack of knowledge in formatting innovations, lack of teaching materials and seminars to learning new teaching techniques are the main contributors. As per Fungo (2015) although teachers already went through excessive trainings, it does not mean that they do not have ineffective habits in teaching language and literature. The researcher recommends recognition of LGBTQ+ teachers in the field of academics, as a basis of the Department of Education and educational agencies. 3P'S program: Pagtanggap at Pagkilala sa kakayahang Pang-akademik ng mga gurong LGBTQ+ was recommended to recognize the innovations, approaches and teaching methodologies of LGBTQ+ teachers in different fields of discipline and Gender and Development program and platforms.

Keywords: gurong lgbtq+ (lesbian, gay, bisexual, transgender and queer teacher), istratehiya (strategies), inobatibong pamamaraan (innovative strategies), impak (impacts), panukalang 3P's (pagtanggap at pagkilala sa kakayahang pang-akademik ng mga gurong LGBTQ+ 


\section{Performans ng mga Gurong Lesbian, Gay, Bisexual, Transgender, Queer (LGBTQ+) sa pagtuturo ng akademikong Filipino}

\section{Introduction}

Ang guro bilang isang instrumento ng pagkatuto ay may malawak na responsibilidad ayon sa kanilang sinumpaang katungkulan. Ang guro ay may isang mataas na kredibilidad sa mata ng mga mag-aaral. Sila ang nagsisilbing pundasyon ng sistematiko at organisadong pagbuo ng mag-aaral sa makabuluhang kaisipan, kaugalian at pagkatao. Alinsunod sa RA 4670 o Magna Carta for Public Teachers, Sek. 2, ang guro ay ang mga indibidwal na saklaw ang pagtuturo sa isang silid-aralan, sa iba't ibang antas ng pagtuturo, sa isang buong kasanayan, maging isang guidance counselor, school librarian, pambokasyunal at industriyal na tagasanay, at iba pang indibidwal na sinasaklaw ang pang-administratibo at superbisyon na pinagtibay ng isang pampublikong kagawaran. Hindi na sakop nito ang mga sumusunod: school nurse, school physician, school dentists at iba pang mga manggagawa.

Hindi lingid sa kaalaman ng lahat ang tanging gampanin ng isang guro sa isang dimensyunal na pagtuturo. Saklaw nito ang iba't ibang larangan ng pagkatuto upang higit na malinang ang kakayahan ng bawat mag-aaral sa aspekto ng pagharap sa mga kasalukuyang kaganapan sa lipunan. Ang guro ay kumakatawan sa isang masusing pagsusuri ng mga kaalaman, pagkatuto, abilidad, kakayahan maging pagkatao ng isang indibidwal. Sa patuloy na pagbabago ng sistema ng lipunan, maraming mga salik ang higit na nakaaapekto at nakaaambag sa paglinang ng kakayahan ng mga mag-aaral sa iba’t ibang larang ng pagtuklas maging sa wika man o panitikan. Mga istilo, pamamaraan, metodo at iba pang teknikal na suporta na naglalayon ng pag-unlad ng kakayahang dimensyunal ng pagkatuto ng isang mag-aaral. Kung higit na susuriin ang tanging layunin ng Kagawaran ng Edukasyon ay lubusang nakatuon sa isang pagkatuto ng mag-aaral, hindi gaanong binibigyan ang pagkakakilanlan ng isang guro sa isang mapamaraan nitong pagkakakilanlan bilang isang natatanging guro sa isang oryentasyong sekswal.

Ayon sa teorya ng Queer (Valocchi, 2005), ito ay pagbibigay ng pagtangin sa kakayahan ng mga homosekswal at heterosekswal sa larangang sosyal at panlipunan. Inilalahad sa teoryang ito ang natatanging kahinaan at kalakasan ng mga homosekswal at heterosekwal sa lipunan. Ang kanilang gampanin at pagkakakilanlan. Kung susuriin ang mga nilalaman ng programa ng Kagawaran, alinsunod sa Gender and Development, nakatuon lamang ito kung paano tatanggapin ng walang pakundangang pag-iimbot ang kakayahan ng mga mag-aaral (Espina, 2007). Hindi pa lubusang nabibigyan ng pansin ang mga gurong nabibilang sa isang natatanging oryentasyon bilang isang guro.

Alinsunod sa panukalang Sexual Orientation and Gender Identity and Expression Equality (SOGIE Bill), binibigyang saklaw ang kakayahan at pantay na pagtingin sa karapatang sekswal sa mga lesbyana, bakla, bisexual, transgender at iba pa. Pinatotohanan nito ang pagkalingang sosyal at panlipunang pagkakakilanlan ng isang indibidwal (Murcia, 2006). Ayon sa alintuntunin ng mga Karapatan Pantao ng Ontario (Ontario Human Rights Code), ang bawat tao ay may karapatan maging Malaya mula sa diskriminasyon batay sa kasarian at kabilang dito ang sekswal na panliligalig. Ipinaliliwanag nito na lahat ng tao maging sa iba’t ibang antas ng pamumuhay o estado sa buhay ay may nakaangklang karapatan na tanggapin at higit na maging pantay ang pagtanggap sa lipunang ng mga taong nabibilang sa ikatlong kasarian o homosekwal (Reyes, 1997). Ayon kay Rep. Geraldine Roman, kauna-unahang transgender Military Officer, "Service knows NO GENDER" (Tan, 2002). Ang kasarian ay hindi hadlang upang maglingkod sa bayan. Walang kasarian ang higit na mangingibabaw upang piliin sa isang katungkulan, lahat ay pantay sa paningin ng lipunan, anumang antas o estado ng pamumuhay ng isang indibidwal. Pinapahayag ng katagang ito na ang kasarian ng isang tao ay isang oryentasyon lamang ng kaniyang personal na pagkakakilanlan, hindi ito magiging batayan ng isang masusing pagpapalawig ng isang pampropesyunal na paglago.

2 Consortia Academia Publishing (A partner of Network of Professional Researchers and Educators) 
Performans ng Gurong Lesbian, Gay, Bisexual, Transgender, Queer (LGBTQ+) sa pagtuturo ng Filipino

Sa patuloy na pakikipag-ugnayan ng mananaliksik sa isang programa ng GAD o Gender and Development, napag-alaman ng mananaliksik na parami na nang parami ang mga indibidwal na nabibilang sa ikatlong kasarian o ang tinatawag na LGBTQ+ na oryentasyon (Veer, 2005) Napag-alamang hindi lamang mga mag-aaral ang nabibilang dito, maging mga tagasanay, propesyonal at guro ay nakararanas ng ganitong pangsekswal na oryentasyon. Batay sa pananaliksik ng pagpapatibay ng suliranin, napag-alaman rin na wala pang higit na mananaliksik ang binibigyang atensyon ang mga gurong nabibilang sa LGBTQ, maging impak ng pagtuturo sa iba't ibang larangan ng pagtuklas sa Wika at Panitikan at pagtuklas ng kakaibang kakayahan ng mga gurong nabibilang sa nasabing oryentasyon sa isang pagtuklas ng karunungan at kasanayan.

Ang mga nabanggit na mga kadahilanan ang nag-udyok sa mananaliksik upang isagawa ang pag-aaral tungkol sa performans ng mga gurong LGBTQ sa pagtuturo ng akademikong Filipino sa larangan ng wika at panitikan.

\subsection{Paglalahad ng suliranin}

Layunin sa pag-aaral na ito na matukoy ang Performans ng mga gurong LGBTQ+ sa pagtuturo ng Wika at Panitikan sa Pamprobinsiyang Sangay ng Sorsogon. (1) Matukoy ang mga istratehiyang ginagamit sa pagtuturo ng Wika at Panitikan. (2) Malaman ang mga salik na nakakaapekto sa pagtuturo ng mga gurong ito. (3) Masuri ang lebel ng Performans ng mga gurong LGBTQ+ sa pagtuturo ng Akademikong Filipino. (4) Malaman ang mga impak ng pagtuturo. (5) Interbensyon na maaaring mabuo mula sa mga nakalap at sinuring datos ng pag-aaral.

\section{Pamamaraang ginamit}

Ginamit ang deskriptib-analisis na disenyo ng pananaliksik. Ang mga naging kalahok ay binubuo ng 1,252 mula sa 22 Pansekundaryang Paaralan sa Una at Ikalawang Pamprobinsiyang Sangay ng Sorsogon na kinakatawan ng mga Gurong LGBTQ+ na nagtuturo ng akademikong Filipino. Gumamit ng talatanungan at tseklist para malaman ang mga istratehiyang ginamit sa pagtuturo ng mga gurong LGBTQ+ sa larangan ng wika at panitikan. Nagsagawa ng pakikipanayan sa mga kalahok sa pamamagitan ng paghingi ng pahintulot at pagpapaliwanag sa isinagawang pananaliksik. Ginamit ang Frequency count, percentage at pagraranggo upang maanalisa ang datos sa impak, salik maging performans ng mga gurong LGBTQ+ sa pagtuturo ng Akademikong Filipino. Purposive Sampling ang ginamit ng mananaliksik sa pagkuha ng 30 Gurong LGBTQ+ na kalahok bilang target ng pananaliksik. Inalam na muna ng mananaliksik ang mga Pansekundaryang Paaralan na mayroong Gurong LGBTQ+ na nagtuturo sa Akademikong Filipino. May iilan na ipinahayag nila ang kanilang sarili ayon sa pagkakaunawa nila sa kanilang sekswal na oryentasyon ngunit may iilan na itinago ang sarili nilang pagkakakilanlan.

Gumamit rin ng Random Sampling sa pagpili ng mga kalahok sa bawat Paaralan ayon lamang sa mga target na kalahok na nabibilang sa komunidad ng LGBTQ+. Ito ay ginamit para sa mga mag-aaral antas Junior Hayskul at Senior Hayskul, kapwa-guro maging sa mga Puno ng Kagawaran. Ang mga nakalap na datos ay inalisa, sinuri at binigyan ng interpretasyon gamit ang pagbilang, at pagraranggo. Kinuha rin ng mananaliksik ang saklaw (range) na performa ng mga gurong LGBTQ+ alinsunod sa IPCRF-RPMS na instrumento na ginagamit ng Kagawaran ng Edukasyon.

\section{Mga natuklasan}

\subsection{Mga istratehiyang ginamit ng mga gurong LGBTQ+ sa pagtuturo ng Wika at Panitikan}

Natuklasan na Ang istratehiyang ginagamit sa pagtuturo ng Akademikong Filipino ng mga Gurong LGBTQ+ sa larangan ng Wika ay Reversal-dereversal na istratehiya na mayroong 304 na kalahok na sumagot na may bahagdang 8.64. Pangalawa sa ranggo ang Pabasang Pamaraan na sinagutan at sinundan ng On-line hybrid courses na may kalahok na 270 na may bahagdang 7.67 na sumagot. Ang mga tuklas o imbentong paraan ng 
Gelilio, E.

pagtuturo ay mahalaga upang higit na mabilis, magaan at epektibo ang pagkatuto (Sandoval, 2015). Kaya’t kailangan magkaroon ng malawak na kaalaman ang guro sa paksang kanyang tinatalakay upang may kakayahan siyang bigyan ng bagong bihis ang paksang tinatalakay ng sa ganun ay mabuksan niya ang mas malikhaing kaisipan ng kanyang mga mag-aaral sa pagkatuto sa Wika.

Nabanggit din ni Jagnaan (2014), pangunahing tungkulin ng guro ang pagtuturo. Ito ay upang magbigay ng impormasyon at gumising sa kawilohan ng mga mag-aaral sa paksang tinatalakay. ang mabuting pangangasiwa ng guro ay pagkakaroon ng episyenteng galaw at mga gawain at pamamahala ng mga gawaing higit na magdudulot sa mag-aaral ng kapaki-pakinabang na karaan.

\section{Talahanayan 1}

Istratehiyang ginagamit sa pagtuturo ng Akademikong Filipino sa larangan ng Wika

\begin{tabular}{ccccccc}
\hline Wika & L+ & G & PK & Kabuuan & Bahagdan & Ranggo \\
\hline Reversal-Dereversal & 30 & 267 & 7 & 304 & 8.64 \\
Cultured Gramatika & 23 & 56 & 5 & 84 & 2.39 \\
On-line hybrid courses & 30 & 234 & 6 & 270 & 7.67 & 10 \\
Games Experiment o Simulation & 17 & 178 & 5 & 200 & 5.68 \\
Audiolingualism & 30 & 178 & 7 & 215 & 6.11 & 6 \\
Clicker use in class & 23 & 165 & 3 & 191 & 5.43 & 4 \\
Paglilipat-baybay (grammar translation) & 22 & 123 & 2 & 147 & 4.18 & 9 \\
Buzz Session & 27 & 145 & 2 & 174 & 4.94 & 8 \\
Pabasang Pamamaraan & 26 & 256 & 8 & 290 & 24 & 2 \\
Interdisciplinary Teaching & 26 & 221 & 5 & 252 & 7.16
\end{tabular}

Pananda: L+.-LGBTQ+ PK-Puno ng Kagawaran G. - Guro

Samantala, ang istratehiyang ginagamit sa pagtuturo ng mga Gurong LGBTQ+ sa larangan ng Panitikan ay Why-Why diagram na sinagutan ng 310 na kalahok na may bahagdang 8.81. Pangalawang istratehiya sa pagtuturo ng Panitikan ang Nepoleonic Technique na may 7.84 na bahagdan ng mga kalahok ang sumagot sa nasabing pag-aaral at sinundan ng DRTA, Directed Reading Thinking Activity na may 273 na kalahok ang sumagot na may bahagdang 7.76. Nabanggit sa pag-aaral ni Bigham (2018), ang mga kabataang LGBTQ+ ay nangangailangan ng gurong LGBTQ+ bilang isang huwaran. Upang maiangat ang mga kabataang LGBTQ+, maraming Gurong LGBTQ+ rin ang kailangan. Pinatutunayan ng pag-aaral na ito na ang mga gurong LGBTQ+ ay nagtuturo upang makuha ang tanging layunin ng mataas na antas ng pagkatuto, sapagkat ang Why-Why Diagram ay hinuhukay ang mataas na karunungan ng mga mag-aaral sa paraan ng pag-aanalaisa ng konsepto sa teksto o panitikang nabasa.

\section{Talahanayan 2}

Istratehiyang ginagamit sa pagtuturo ng Akademikong Filipino sa larangan ng Panitikan

\begin{tabular}{|c|c|c|c|c|c|c|}
\hline Panitikan & $\mathbf{L}+$ & $\mathbf{G}$ & PK & Kabuuan & Bahagdan & Ranggo \\
\hline Inverse Brainstorming & 30 & 222 & 7 & 259 & 7.38 & 4 \\
\hline Fresh Eye & 12 & 198 & 9 & 219 & 6.22 & 8 \\
\hline Nepoleonic Technique & 16 & 249 & 11 & 276 & 7.84 & 2 \\
\hline Why-Why Diagram & 30 & 267 & 13 & 310 & 8.81 & 1 \\
\hline DRTA, Directed Reading Thinking Activity & 30 & 231 & 12 & 273 & 7.76 & 3 \\
\hline Story Frame (Pakuwadradong Pagkukuwento) & 24 & 199 & 12 & 235 & 6.68 & 6 \\
\hline Fan Fact Analyzer & 30 & 167 & 15 & 212 & 6.02 & 9 \\
\hline Creative Imaging & 26 & 200 & 17 & 243 & 6.90 & 5 \\
\hline Photo Excursion & 12 & 125 & 11 & 148 & 4.20 & 10 \\
\hline $\begin{array}{l}\text { KWL, What I Know, What I want to find out, What I } \\
\text { learned? }\end{array}$ & 29 & 178 & 18 & 225 & 6.38 & 7 \\
\hline
\end{tabular}

3.2 Mga Salik na nakaapekto sa pagtuturo ng Akademikong Filipino ng mga urong LGBTQ+ sa larangan ng Wika at Panitikan

Ang mga salik na nakaaapekto sa pagtuturo ng Akademikong Filipino ng mga Gurong LGBTQ+ sa larangan

4 Consortia Academia Publishing (A partner of Network of Professional Researchers and Educators) 
Performans ng Gurong Lesbian, Gay, Bisexual, Transgender, Queer (LGBTQ+) sa pagtuturo ng Filipino

ng Wika ay ang kawalan ng inobatibong pamamaraan ng pagtuturo na may 329 na kalahok ang sumagot na may bahagdang 9.35. Pangalawang salik ang kakulangan ng mga istratehiya at pamamaraang pangwika na may $292 \mathrm{o}$ 8.30 bahagdan ng kalahok ang sumagot at sinundan ng walang sapat na kagamitang pampagkatuto bilang salik ng pagtuturo ng Wika ng mga Gurong LGBTQ+ ayon sa 291 na kalahok na sumagot na may bahagdang 8. 27.

Pinatunayan sa pag-aaral ni Fungo (2015), bagaman ang guro ay dumaan na sa maraming pagsasanay hindi pa rin maalis na may pamamaraang hindi pa rin kawili-wili lalo na sa pagtuturo ng wika at panitikan. Kinakailangan na ang mga guro ay nabibigyan ng panahon para maitala ang kanilang mga bagong-tuklas upang ipakilala rin sa iba pang disiplina at pagkatuklas. Banggit nga sa pag-aaral ni Kizlik (banggit ni Manallo, 2016), sa paggamit ng teknik sa gawaing pagtuturo, magiging epektibo lamang ito kung nauunawaan ng guro ang pangunahing panuntunan at mga palagay kung saan at paano iaangkop ang isang istratehiya sa pagtuturo. Mahalaga ayon sa kaniya na magkaroon ng sapat na kagamitan sa pagtuturo maging mga bagong tuklas na pamamaraan at dulog ng pagkatuto.

\section{Talahanayan 3}

Salik na nakakaapekto sa pagtuturo ng mga gurong LGBTQ+ sa Akademikong Filipino sa Larangan ng Wika

\begin{tabular}{|c|c|c|c|c|c|c|}
\hline Wika & L+ & $\mathbf{G}$ & PK & Kabuuan & Bahagdan & Ranggo \\
\hline Kakulangan ng mga istratehiya at pamamaraang pangwika & 30 & 245 & 17 & 292 & 8.30 & 2 \\
\hline Walang sapat na kagamitang pampagkatuto & 29 & 256 & 6 & 291 & 8.27 & 3 \\
\hline $\begin{array}{l}\text { Kakulangan sa panahon upang higit na maunawaan ang wika } \\
\text { at gramatika }\end{array}$ & 26 & 210 & 16 & 252 & 7.16 & 4 \\
\hline $\begin{array}{l}\text { Walang sapat na leksikon upang maging batayang } \\
\text { panggramatika ng mga mag-aaral }\end{array}$ & 27 & 178 & 12 & 217 & 6.16 & 7 \\
\hline Walang inobatibong pamamaraan ng pagtuturo & 21 & 289 & 19 & 329 & 9.35 & 1 \\
\hline Kakulangang panggramatikang kasanayan & 29 & 178 & 11 & 218 & 6.19 & 6 \\
\hline Hindi nakaangkla ang mga gurong nagtuturo sa wika & 21 & 173 & 8 & 202 & 5.74 & 8 \\
\hline Walang sapat na pagsasanay sa mga gurong LGBTQ+ & 30 & 200 & 16 & 246 & 6.99 & 5 \\
\hline $\begin{array}{l}\text { Hindi pagtanggap ng institusyon sa pansariling } \\
\text { pagkakakilanlan ng gurong nabibilang sa ikatlong kasarian }\end{array}$ & 8 & 45 & 2 & 55 & 1.56 & 10 \\
\hline $\begin{array}{l}\text { Kakulangan ng mga gurong magtuturo ng Akademikong } \\
\text { Filipino }\end{array}$ & 19 & 100 & 12 & 131 & 3.72 & 9 \\
\hline
\end{tabular}

Pananda: L+.-LGBTQ+ PK-Puno ng Kagawaran G. - Guro

Samantala, ang salik na nakaaapekto sa pagtuturo ng Panitikan sa Akademikong Filipino ng mga Gurong LGBTQ+ ay ang hindi sapat na pagsasanay o palihan sa pagtuturo ng Panitikan na may 208 na kalahok ang sumagot o 5.91 ayon sa bahagdang pag-aanalisa. Pangalawang salik ang hindi sapat na oras upang basahin ang mga akdang pampanitikan na may 204 o 5.80 na bahagdan ng kalahok ang sumagot at sinundan ng walang inobatibong pagtuturong naisagawa sa panitikan na may 192 kalahok na sumagot kaakibat ang 5.45 na bahagdan. Binanggit sa pag-aaral ni Veer (2015), ang pag-unlad ng isang bansa ay nakasalalay sa kalidad ng mga guro, at ang mga pagsasanay ay mahalaga para sa mga guro. Ang mga "trained" na guro ay mas maraming magagawa kaysa sa mga "untrained" na guro. Kailangan ng mga guro ang patuloy na pag-unlad sa larangan ng edukasyong upang mapaunlad din ang pagtuturo at pagkatuto sa paaralan. Ayon rin kay Sandoval (2015), ang mga tuklas o imbentong paraan ng pagtuturo ay mahalaga upang higit na mabilis, magaan at epektibo ang pagkatuto. Binanggit rin sa kaniyang pag-aaral na maging inobatibo ang mga guro lalong-lalo na sa pagtuturo ng iba’t ibang disiplina at larangan.

Pinatutunayan ng resultang ito na kinakailangan na magsagawa ng mga bagong tuklas na pamamaraan maging istratehiya ng pagtuturo lalong-lalo na sa Wika at Panitikan sapagkat hindi madali ang binabalikat ng isang guro lalong-lalo na sa mga Gurong LGBTQ+ na nagtuturo sa akademikong Filipino.

\subsection{Lebel ng Performans ng mga gurong LGBTQ+ sa pagtuturo ng Akademikong Filipino}

Ang lebel ng performans ng mga Gurong LGBTQ+ sa pagtuturo ng Akademikong Filipino ay nasa interpretasyon ng VS (VERY SATISFACTORY) alinsunod sa DepEd Order No. 42, s.2017 na may 4.32 bilang kabuuang saklaw o range ng Performans ng mga gurong LGBTQ+ sa loob ng sunod-sunod na taon ng panuruan 
Gelilio, E.

2016-2019. 4.23 ang range ng performans ng mga Gurong nabibilang sa sekswal na oryentasyon bilang Gay na may interpretasyong VS (VERY SATISFACTORY) sa 6 na kalahok, 4.30 ang range naman sa mga gurong nabibilang sa oryentasyong Bisexual na may interpretasyong VS (VERY SATISFACTORY) sa 4 na kalahok samantalang 4.30 rin ang range ng performans ng mga gurong nabibilang sa Queer na sekswal na oryentasyon na may 20 kalahok.

\section{Talahanayan 4}

Salik na nakakaapekto sa pagtuturo ng mga gurong LGBTQ+ sa Akademikong Filipino at sa Panitikan

\begin{tabular}{|c|c|c|c|c|c|c|}
\hline Panitikan & $\mathbf{L}+$ & G & PK & $\begin{array}{c}\text { Kabuua } \\
\text { n }\end{array}$ & Bahagdan & Ranggo \\
\hline Hindi pagkilala sa mga local na akda & 25 & 10 & 89 & 124 & 3.52 & 6 \\
\hline $\begin{array}{l}\text { Hindi sapat na pagsasanay at palihan sa pagtuturo ng } \\
\text { panitikan }\end{array}$ & 30 & 11 & 167 & 208 & 5.91 & 1 \\
\hline $\begin{array}{l}\text { Kakulangan ng oras sa pagbibigay ng katuturan sa mga } \\
\text { salitang tekstwal }\end{array}$ & 26 & 14 & 78 & 118 & 3.35 & 7 \\
\hline Kakulangan ng mga dulog at pamamaraan ng pagtuturo & 30 & 18 & 143 & 191 & 5.43 & 4 \\
\hline Walang inobatibong pagtuturo na naisagawa sa panitikan & 30 & 17 & 145 & 192 & 5.45 & 3 \\
\hline $\begin{array}{l}\text { Hindi malinaw na pagpapakilala sa mga akdang } \\
\text { pampanitikan sa ibang karatig lugar ng Pilipinas }\end{array}$ & 14 & 11 & 78 & 103 & 2.93 & 9 \\
\hline $\begin{array}{l}\text { Apektado ang sariling pagkakakilanlan sa pagtuturong } \\
\text { moral ng panitikan }\end{array}$ & 5 & 7 & 76 & 88 & 2.50 & 10 \\
\hline $\begin{array}{l}\text { Pagsasaayos ng istukturang pampanitikan, haba at } \\
\text { panahong kinabibilangan }\end{array}$ & 24 & 1 & 89 & 114 & 3.24 & 8 \\
\hline Kakulangan ng mga babasahing pampanitikan & 26 & 13 & 123 & 162 & 4.60 & 5 \\
\hline $\begin{array}{l}\text { Hindi sapat na oras upang basahin ang mga akdang } \\
\text { pampanitikan }\end{array}$ & 27 & 7 & 170 & 204 & 5.80 & 2 \\
\hline
\end{tabular}

Alinsunod sa DepEd Order No. 42, s. 2017, "National Adoption and Implementation of the Philippine Professional Standard for Teachers", kinakailangan na maging holistic ang isang guro sa paraan ng kaniyang pagtuturo. Isinaad rin sa batas na ito na maging dimensyunal na guro ang lahat ng mga nagtuturo upang higit na makuha ang layunin ng edukasyon tiyak sa mga disiplina at iba pang aspekto ng pagkatuto. Malinaw na nakasaad din sa Artikulo IV, Seksyon 2 "Code of Ethics", ang bawat guro ay nararapat na magtataguyod ng pagkakaroon ng pinakamataas na istandard sa kalidad ng edukasyon, gagawa ng pinakamahusay na paghahanda para sa tungkulin ng pagtuturo, at inaasahang magiging pinakamahusasy sa pagsasakatuparan ng mga gawaing kaugnay ng kaniyang propesyon sa lahat ng panahon.

Pinatutunayan na ang pagtuturo ay hindi lamang nagaganap sa kung ano ang maibibigay ng guro bagkus kung paano higit na malilinang ang kakayahang dimensyunal na mag-aaral.

\section{Talahanayan 5}

Deskripsiyon ng Performans batay sa PPST-RPMS na instrumento ng Kagawaran ng Edukasyon

\begin{tabular}{cc}
\hline Saklaw (range) & Interpretasyon (Adjectival Rating) \\
\hline $\mathbf{4 . 5 0 0 - 5 . 0 0 0}$ & Outstanding (O) \\
$\mathbf{3 . 5 0 0 - 4 . 4 9 9}$ & Very Satisfactory (VS) \\
$\mathbf{2 . 5 0 0 - 3 . 4 9 9}$ & Satisfactory (S) \\
$\mathbf{1 . 5 0 0 - 2 . 4 9 9}$ & Unsatisfactory (US) \\
Pababa 1.499 & Poor (P) \\
\hline
\end{tabular}

Alinsunod sa DepEd Order No. 42, s. 2017, "National Adoption and Implementation of the Philippine Professional Standard for Teachers", ang instrumentong ito ang gagamitin sa lahat ng mga propesyunal na empleyado ng Kagawaran ng Edukasyon sa iba't ibang disiplina. Magiging standard ang pag-alam sa antas maging lebel ng performans ng mga guro tungo sa tanging layunin ng Edukasyon. Nakabatay rin rito ang Results-based Performance Management System (RPMS) upang higit na masukat ang kakayahang pampropesyunal ng mga guro. Ginamit ito upang higit na makita ang lebel ng performans ng mga gurong LGBTQ+ sa pagtuturo ng akademikong Filipino antas sekundarya sa loob ng taong panuruan 2016-2019.

6 Consortia Academia Publishing (A partner of Network of Professional Researchers and Educators) 


\section{Talahanayan 6}

Lebel ng Performans ng mga Gurong LGBTQ+ sa loob Taong Panuruan 2016-2019

\begin{tabular}{|c|c|c|c|c|c|c|c|}
\hline Gurong LGBTQ+ & 2016-2017 & $2017-2018$ & $2018-2019$ & $\mathrm{~S}$ & I & KS & KI \\
\hline $\mathrm{G} 1$ & 4.53 & 4.50 & 4.50 & 4.51 & $\mathrm{O}$ & 4.23 & VS \\
\hline G2 & 4.56 & 4.50 & 4.60 & 4.55 & $\mathrm{O}$ & & \\
\hline G3 & 4.35 & 4.51 & 4.52 & 4.46 & VS & & \\
\hline G4 & 4.05 & 4.35 & 4.56 & 4.32 & VS & & \\
\hline G5 & 3.89 & 4.01 & 4.60 & 4.17 & VS & & \\
\hline G6 & 4.50 & 4.52 & 4.57 & 4.53 & $\mathrm{O}$ & & \\
\hline B1 & 4.45 & 4.47 & 4.50 & 4.47 & VS & 4.30 & VS \\
\hline B2 & 3.89 & 3.78 & 4.30 & 3.99 & VS & & \\
\hline B3 & 4.50 & 4.54 & 4.58 & 4.54 & $\mathrm{O}$ & & \\
\hline B4 & 3.90 & 4.30 & 4.30 & 4.17 & VS & & \\
\hline Q1 & 4.56 & 4.45 & 4.50 & 4.50 & $\mathrm{O}$ & 4.30 & VS \\
\hline Q2 & 3.90 & 4.25 & 4.58 & 4.24 & VS & & \\
\hline Q3 & 4.00 & 4.35 & 4.50 & 4.28 & VS & & \\
\hline Q4 & 3.58 & 3.59 & 4.50 & 3.89 & VS & & \\
\hline Q5 & 4.30 & 4.60 & 4.76 & 4.55 & $\mathrm{O}$ & & \\
\hline Q6 & 3.56 & 3.50 & 3.98 & 3.68 & VS & & \\
\hline Q7 & 4.56 & 4.55 & 4.50 & 4.54 & $\mathrm{O}$ & & \\
\hline Q8 & 3.90 & 3.78 & 4.30 & 3.99 & VS & & \\
\hline Q9 & 4.36 & 4.45 & 4.44 & 4.42 & VS & & \\
\hline Q10 & 3.89 & 3.67 & 4.50 & 4.02 & VS & & \\
\hline Q11 & 4.58 & 4.63 & 4.51 & 4.57 & $\mathrm{O}$ & & \\
\hline Q12 & 3.89 & 3.90 & 4.50 & 4.10 & VS & & \\
\hline Q13 & 4.20 & 4.23 & 4.54 & 4.32 & VS & & \\
\hline Q14 & 3.57 & 3.88 & 4.12 & 3.86 & VS & & \\
\hline Q15 & 4.50 & 4.70 & 4.45 & 4.55 & $\mathrm{O}$ & & \\
\hline Q16 & 4.00 & 4.56 & 4.35 & 4.30 & VS & & \\
\hline Q17 & 3.98 & 4.03 & 4.06 & 4.02 & VS & & \\
\hline Q18 & 4.67 & 3.99 & 4.62 & 4.43 & VS & & \\
\hline Q19 & 4.67 & 4.56 & 4.58 & 4.60 & $\mathrm{O}$ & & \\
\hline Q20 & 4.56 & 4.44 & 4.51 & 4.50 & $\mathrm{O}$ & & \\
\hline
\end{tabular}

Kabuuang saklaw at interpretasyon ng mga gurong LGBTQ+

\subsection{Impak ng pagtuturo ng gurong $L G B T Q+$ sa mga mag-aaral}

Ang Positibong impak ng pagtuturo ng isang LGBTQ+ na guro sa akademikong Filipino ay mabisa ang pamamaraan ng pagtuturo sa paraang kaaya-aya at masaya na sinagutan ng 855 na kalahok o 9.50 na bahagdan. Pangalawa ang madaling maunawaan ng mag-aaral ang paksa na sinagutan ng 832 na kalahok o 9.24 na bahagdan at sinundan ng magiliw ang daloy ng pagtuturo na sinagutan ng 756 na kalahok o 8.40 na bahagdan. Binanggit sa pag-aaral nina Carrington at Skelton (2003), nagkakaroon ng kakintalan sa pag-aaral ang mga mag-aaral kung ang kanilang guro ay may kaugnayan sa kanilang sekswal na oryentasyon. Nabanggit rin sa pag-aaral na ang mga gurong nabibilang rito ay may angking kasangkapan ng kasiyahan at kagiliwan sa pagtuturo ng araling pandisiplina. Pinatutuhanan nito na likas na sa mga gurong LGBTQ+ ang kakayahang magbigay saya at magpangiti sa oras ng klase maging sa ibang aspekto ng pagkatuto. Pinatutunayan na ang isang indibidwal na nabibilang sa ikatlong kasarian ay may katangi-tanging talento upang higit na mapadali ang anumang kahirapang kinahaharap ng lipunan, kung ipapasok ito sa paraang pagtuturo, ang mga gurong nabibilang dito ay may angking kakayahan upang higit na payabungin at pagyamanin ang pagtuturo sa disiplinang kanilang tinuturuan.

Samantala, ang negatibong impak ng pagtuturo ng mga gurong LGBTQ+ ay sinagutan ng 627 na kalahok na may bahagdang 6. 97 na nagkakaroon ng sekswal na ugnayan sa mag-aaral partikular sa institusyong kinabibilangan. Pangalawang negatibong impak na sinagutan ng 374 na kalahok na may 4.16 na bahagdan na ang mga gurong LGBTQ+ ay walang inobasyon sa pamamaraan ng pagtuturo at sinundan ng hindi sapat ang 
Gelilio, E.

kaalaman sa pagtuturo ng balarila at pampanitikan na may 3.44 na bahagdan ng kalahok ang sumagot. Napatunayan nga sa pag-aaral ni Coprado (2006), ang mga gurong nabibilang sa homosekswalidad ay isang malaking usapin ng paaralan maging sa impak ng mga mag-aaral. Sila ang mga taong hindi dapat na pagkatiwalaan at kadalasang nagiging suliranin pagdating sa respeto at dignidad.

\section{Talahanayan 7}

Positibong Impak ng pagtuturo ng gurong LGBTQ+ sa Akademikong Filipino

\begin{tabular}{|c|c|c|c|c|c|}
\hline Positibo & JHS & SHS & Kabuuan & Bahagdan & Ranggo \\
\hline Magiliw an daloy ng Pagtuturo & 400 & 356 & 756 & 8.40 & 3 \\
\hline $\begin{array}{l}\text { Maenganyo ang mga mag-aaral na makiisa sa talakayan ng } \\
\text { Klase }\end{array}$ & 396 & 267 & 663 & 7.37 & 5 \\
\hline $\begin{array}{l}\text { Mapaunlad ang intelektwal na kakayahan ng mag-aaral sa } \\
\text { Akademikong Filipino }\end{array}$ & 234 & 200 & 434 & 4.82 & 10 \\
\hline Madaling maunawaan ng mag-aaral ang paksa & 434 & 398 & 832 & 9.24 & 2 \\
\hline $\begin{array}{l}\text { Inspirasyon ng mga mag-aaral sa patuloy na } \\
\text { pakikipag-ugnayan sa karunungan ng Akademikong Filipino }\end{array}$ & 345 & 231 & 576 & 6.40 & 6 \\
\hline $\begin{array}{l}\text { Mabisa ang pamamaraan ng pagtuturo sa paraang kaaya-aya } \\
\text { at masaya }\end{array}$ & 432 & 423 & 855 & 9.50 & 1 \\
\hline $\begin{array}{l}\text { Gumagamit ng mga larong pangwika at pampanitikan upang } \\
\text { malinang ang kakayahang pangkarunungan }\end{array}$ & 321 & 234 & 555 & 6.17 & 9 \\
\hline $\begin{array}{l}\text { Gumagawa ng mga Inobatibong pamamaraan ng pagtuturo } \\
\text { ng wika at panitikan }\end{array}$ & 267 & 290 & 557 & 6.19 & 8 \\
\hline $\begin{array}{l}\text { Binibigyang pansin ang kahinaan ng mga mag-aaral sa } \\
\text { Akademikong Filipino }\end{array}$ & 343 & 369 & 712 & 7.91 & 4 \\
\hline $\begin{array}{l}\text { Ipinapasok ang sarili sa karanasang global at modern sa } \\
\text { pagkatuto at paglinang ng karunungan panggramatika sa } \\
\text { mga mag-aaral }\end{array}$ & 300 & 267 & 567 & 6.30 & 7 \\
\hline
\end{tabular}

Mahihinuha sa pag-aaral na ito batay sa mga datos na nakalap na ang mga gurong LGBTQ+ ay nagkakaroon ng sekswal na pag-aabuso o ugnayan sa mga mag-aaral, ito marahil ang nagiging dahilan kung bakit karamihan sa mga mag-aaral ay naabuso rin ang kakayahang pagtuturo ng mga gurong LGBTQ+ dahil sa kawalan ng gap sa dalawang aspekto ng pagkatuto. Ngunit ito ay sumasalungat sa sinambit ni Congw. Geraldine B. Roman (2019) ng Unang Distrito ng Bataan, ang kauna-unahang transgender na pulitiko sa bansa, dahil ang mga miyembro ng LGBT ay tao, dapat silang irespeto at itrato bilang tao at hinihingi lamang nila ay patas na tinatamasa rin ng karamihan anuman ang kanilang sekswal na oryentasyon at pangkasariang pagkakakilanlan.

\section{Talahanayan 8}

Negatibong Impak ng pagtuturo ng gurong LGBTQ+ sa Akademikong Filipino

\begin{tabular}{|c|c|c|c|c|c|}
\hline Negatibo & JHS & SHS & Kabuuan & Bahagdan & Ranggo \\
\hline Naaabuso ang kakayahang intelektwal ng mga mag-aaral & 5 & 45 & 50 & 0.56 & 10 \\
\hline $\begin{array}{l}\text { Tradisyunal ma pamamaraan ng pagtuturo ang ipinapamalas sa loob } \\
\text { ng pangkarunungang senaryo }\end{array}$ & 35 & 156 & 191 & 2.12 & 8 \\
\hline Laging huli kung pumasok sa oras ng klase & 56 & 78 & 134 & 1.49 & 9 \\
\hline $\begin{array}{l}\text { Gumagamit ng mga salitang nakakasakit upang ibaba ang } \\
\text { kakayahang pangnilalaman ng mga mag-aaral }\end{array}$ & 123 & 167 & 290 & 3.22 & 4 \\
\hline $\begin{array}{l}\text { Hinahayaan lamang na lagging mag-aaral ang umuulat sa unahan na } \\
\text { walang gabay na paraan maging sa aspekto ng pagtuklas ng } \\
\text { pagkatuto }\end{array}$ & 34 & 189 & 223 & 2.48 & 5 \\
\hline $\begin{array}{l}\text { Nagkakaroon ng sekswal na ugnayan sa mag-aaral partikular sa } \\
\text { institusyong tinuturuan }\end{array}$ & 260 & 367 & 627 & 6.97 & 1 \\
\hline $\begin{array}{l}\text { Hindi bukas ang ugnayang pampagtuturo sa mga mag-aaral. } \\
\text { Guro-mag-aaral, mag-aaral-guro }\end{array}$ & 145 & 54 & 199 & 2.21 & 7 \\
\hline Walang inobasyon sa pamamaraan ng pagtuturo & 143 & 231 & 374 & 4.16 & 2 \\
\hline Hindi sapat ang kaalaman sa pagtuturo ng balarila at pampanitikan & 165 & 145 & 310 & 3.44 & 3 \\
\hline $\begin{array}{l}\text { Hindi ipinapasok ang e-learning na istilo upang higit na mabigyang } \\
\text { pansin ng iba't ibang karanasan ng mga mag-aaral sa pagkatuto sa } \\
\text { wika at panitikan }\end{array}$ & 132 & 68 & 200 & 2.22 & 6 \\
\hline
\end{tabular}

8 Consortia Academia Publishing (A partner of Network of Professional Researchers and Educators) 


\subsection{Interbensyon batay sa resulta ng pag-aaral}

Isang panukalang 3P's: Pagtanggap at Pagkilala sa kakayahang Pang-akademik ng mga gurong LGBTQ+ upang maging batayan ng Kagawaran ng Edukasyon maging ang ahensiyang nagbibigay pokus sa Gender and Development na programa at panukala at isang magasing "dalumatrehiya" na kinapapalooban ng mga inobatibong pamamaraan ng pagtuturo ng mga gurong LGBTQ+ sa pagtuturo ng wika at panitikan sa akademikong Filipino. Maaaring pagbatayan ang pananaliksik na ito ng mga susunod na mananaliksik upang higit na palawigin ang pag-aaral sa mga gurong LGBTQ+ ayon sa tunguhin ng susunod na mananaliksik.

\section{Kongklusyon}

Batay sa natuklasan, nabuo ang sumusunod na kongklusyon:

$>$ Iba't ibang istratehiya ang ginagamit sa pagtuturo ng wika at panitikan ang ginagamit ng mga LGBTQ+ sa pagtuturo ng akademikong Filipino sapagkat iba-iba ang pamamaraang ginagamit sa pagtuturo ng akademikong Filipino sa magkaibang larangan ng wika at panitikan.

> May mga salik na nakakaapekto sa pagtuturo ng mga gurong LGBTQ+ sa akademikong Filipino sa larangan ng wika at panitikan.

$>$ Very satisfactory (VS) ang lebel ng performans ng mga gurong LGBTQ+ sa pagtuturo sa akademikong Filipino. Pinatutunayan na mataas ang lebel ng performans tungo sa mabisa at epektibong pagtuturo.

$>$ May positibo at negatibong impak ang pagtuturo ng isang LGBTQ+ na guro sa akademikong Filipino sa larangan ng wika at panitikan.

$>\quad$ Nabuo ang isang panukalang gawain upang mas maituro pa nang may kahusayan ang wika at panitikan at mapanatili ang mataas na lebel ng performans ng mGa gurong LGBTQ+ ng pagtuturo sa akademikong Filipino.

\subsection{Rekomendasyon}

Batay sa mga resulta at kongklusyon ng pag-aaral na ito, ang sumusunod na mga rekomendasyon ay nabuo:

> Bigyan ng pansin ang mga gurong LGBTQ+ sa kanilang pagtuturo ng akademikong Filipino at bigyan ng pagkilala ang mga natatanging kakayahan ng mga gurong ito na makapagtuklas ng mag bagong pamamaraan o inobatibo sa pagtuturo ng larangan ng wika at panitikan.

> Magkaroon ng mga salingkurang-pagsasanay sa mga gurong LGBTQ+ kung paano maisasatitik ang mga inobatibong pamamaraan ng pagtuturo sa larangan ng wika at panitikan.

> Pataasin ang antas ng kaalaman ng mga gurong LGBTQ+ sa pagtuturo ng akademikong Filipino sa pamamagitan ng bukas na pahihintulot sa mga ito na makapagdalo sa iba't ibang lebel ng palihan upang higit na mahubog ang kakayahang pampropesyunal na aspekto ng pagtuturo.

> Magkaroon ng GAD o Gender and Development na oryentasyon sa mga gurong LGBTQ+ upang higit na makilala ang tunay nilang pagkakakilanlan maging tamang pakikipag-ugnayan sa mga mag-aaral sa paraang walang sekswal na interaksyon.

$>\quad$ Kung maaari ay gamitin ang panukalang 3P'S: Pagtanggap at Pagkilala sa kakayahang Pang-akademik ng mga gurong LGBTQ+, upang maging batayan ng Kagawaran ng Edukasyon maging ang ahensiyang nagbibigay pukos sa Gender and Development na programa at panukala. 
Gelilio, E.

$>\quad$ Maaring pagbatayan ang pananaliksik na ito ng mga susunod na mananaliksik upang higit na palawigin ang pag-aaral sa mga gurong LGBTQ+ ayon sa tunguhin ng susunod na mananaliksik.

\section{Talasanggunian}

Aguda, L. A., Tamayo, R., \& Leoncion, B. (2013). Effects off heritage tourism to the municipality of Taal, Batangas, Philippines.

Alamer, R. L. (2015). Effects of discussion-interaction method on the performance of grade IV pupils in Science [Unpublished master's thesis]. Sorsogon State College, Sorsogon City.

Almario, V. S. (2015). KWF Manwal sa Masinop na pagsulat (5th ed.). Komisyon ng Wikang Filipino.

Angara, B. J. (n.d.). House Bill 7165 "Lesbian Gay Rights Act of 1999”.

Arambuyong, J. E. (2018). Istilo sa Pagtuturo ng Balarila at Panitikang Filipino [Unpublished master's thesis]. Sorsogon State College, Sorsogon City.

Bramwell, B., \& Lane, B. (2010). Sustainable tourism and the evolving roles of government planning. Journal of Sustainable Tourism, 18(1), 1-5. https://doi.org/10.1080/09669580903338790

Bucjan, E. R. (n.d.). International Journal of English literature and social sciences.

Espina, L. (2007). Modyul Sa Filipino 1: Komunikasyon Sa akademikong Filipino. Mindshapers Co., Inc.

Gebhard, J. (2006). Teaching English as a foreign or second language, second edition. https://doi.org/10.3998/mpub.147778

House Bill No. 258 “An Act of Prohibiting Discrimination based on Sexual Orientation and Gender Identity and Expression (SOGIE EQUALITY ACT). (n.d.).

Huevos, M. M. (2015). Laro: Isang Estratehiya sa Pagtuturo ng Numeracy [Unpublished master's thesis]. Sorsogon State College, Sorsogon City.

Lucas, R. D. (2008). Mga mabisang Istratehiya sa pagtuturo ng Panitikan sa mga mag-aaral ng Kolehiyo [Unpublished master's thesis]. Bulacan State University, City of Malolos.

Mejia, R. C., Fertijo, B., Borbon, J., \& Barlan, L. (2013). Involvement of the community in promoting marine biodiversity as tourist attraction. Journal of International Academic Research for Multidisciplinary.

Pagkalinawan, L. (2015). Ang tradisyunal at makabagong paraan ng pagtuturo.

Villanueva, V. M. (n.d.). Wikang Filipino: Wikang panlahat, salalayan sa pagtatamo ng likas-kayang pag-unlad, 1-6.

Zafra, G. (2016). Ang pagtuturo ng wika at kulturang Filipino sa disiplinang Filipino (Konteksto Ng K-12). Katipunan: Journal ng mga ng mga Pag-aaral sa Wika, Panitikan, Sining at Kulturang Filipino, 1, 4-28. https://doi.org/10.13185/kat2016.00102 